\title{
Is Point of Care Ultrasound (PoCUS) integrated in clinical exam a key-tool for the future emergency physician?
}

\author{
Adela Golea
}

Emergency Department, University Of Medicine and Pharmacy “Iuliu Haţieganu” Cluj-Napoca, Romania

Emergency field is a difficult area of medical assessment and also a part of high risk of medical errors. For some time physicians have tried to improve the diagnosis and treatment methods using many strategies and devices. The new "sonostethoscope" offers the opportunity to push the imagistic examination in the front line of the clinical examination and patients' monitoring.

The question is how this old medical diagnosis procedure can become more useful for emergencies assessment and clinical decisions?

In the daily practice it is amazing how an ultrasound examination can change the attitude and save life. It is hard to understand when a trauma patient with shock comes in the Emergency Department (ED) and on FAST (Focused assessment with sonography for trauma) and RUSH (Rapid Ultrasound for Shock and Hypotension) examination we identify a pulmonary embolism as the cause of the accident and of the shock status and it might be the cause of death if we do not reach to this diagnosis.

PoCUS has not yet become a standardized examination tool for emergency physicians or practitioners who work in emergency settings, but its utility cannot be ignored. PoCUS is not a replacement of ultrasound studies performed by radiologists or cardiologists. PoCUS should be used to give answers to specific questions in real time, like in respiratory failure cases, to make the

Received Accepted

Med Ultrason

2017, Vol. 19, No 3, 250-251

Corresponding author: Adela C. Golea

Emergency Department,

County Emergency University Hospital

3-5 Clinicilor Street

400006 Cluj-Napoca, Romania

Phone: 0040264431879/1099

Fax: 0040264599637

E-mail: Adela.Golea@umfcluj.ro difference between the "wet" or "dry" lung, which could be the argument in choosing diuretic treatment.

PoCUS is a useful imaging technique for the young emergency physician in front of the "sick patient" with no specific clinical picture. New studies introduced the concept of "supporting role of integrated ultrasound (US) exam" in the diagnosis and the clinical decisions of improving patients flow and also to avoid errors [1]. The great problem in emergency is to take a rapid decision with a lower rate of errors, which could make an optimal flow of patients possible. In terms of good practice we have to balance time, guidelines based quality of care and evidence based practice[2]. Practitioners usually work with hypothetical-deductive models to develop a strategy for proper decision making in order to reach the correct diagnosis and to choose the correct therapy. But the "visual medicine" based on US examination has become an opportunity to offers a new ability for practitioners to check the clinical picture and to avoid making decisional errors [3].

New "sonostethoscope" can improve the emergency clinical practice at any level [4,5], offering the possibility to use PoCUS examination on each step of the diagnosis step of the patients' flow:

1. Prehospital area: a. Identify patients with immediatel life threatening causes and perform an objective triage of red/yellow emergencies; b. Assess emergencies procedures and offer support for monitoring the effects of therapy; c. Reassess the patients hemodynamic status during the transport;

2. ED area: a. Recheck the immediate life threatening situations and notice the differences between critical and noncritical emergencies; $b$. Start the initial transversal evaluation of critical patients and decide the best early therapy for these patients; $c$. 
Identify potential reversible causes of cardiac arrest or shock status; $d$. Monitor the hemodynamic status of critical patients and appreciate the collections, functional activity of heart and lung, and IVC collapse during the treatment administration; e. Assess the procedures and monitor the immediate complications to avoid the errors associated to delayed therapy; f. Recognize the coexisting diagnosis or the appearance of a new acute process which could be a cause of the treatment resistance.

The goal-directed PoCUS examination can be performed in a few minutes, and is generally combined with clinical critical approach of the whole body examination of the critically ill patient. In this way the presenting symptoms become ultrasonographic signs of diagnosis and offer the possibility to take fast clinical decisions to transfer the patients to a regional hospital or to give dispositions to choose immediately a specific therapy, thus avoiding unnecessary radiation exposure.

In our days, when "sick patient" represents greater challenges for the medical practice, it is important to create a model for teaching PoCUS ultrasound or "bedside ultrasound" to improve hospital outcomes [6]. Professional associations of the emergency physicians and other specialties practitioners in the critical field area created a World Interactive Network Focused On Critical UltraSound (WINFOCUS). This scientific network has committed to develop Point-of-Care Ultrasound practice, research, education, technology, and networking, addressing the patients' needs, institutions, services, and communities living in out-of-hospital and in-hospital critical scenarios [7].

Considering US a "key-tool" for clinical making decision, the emergency health care strategy can be improved in terms of early diagnosis with less errors, optimal and urgent therapy, also with a rapid flow of patients in ED.
Improving the emergency assessment, independent of the degree of emergency, with a noninvasive diagnosis and monitoring procedures, like US, provides additional information that supports the work of emergency physician and offers a better quality of care in emergency care situations.

By building a structured PoCUS examination program for practitioners working in emergency settings, we can improve clinical assessment and also monitor the management of critical and noncritical emergencies. Ultrasound examination can offer a "key-tool" to avoid many errors that appear in case of pathologies without clinical manifestations.

\section{References}

3. Medd D. Letter to the Editor: Bedside Ultrasound: Part of the Physical Examination. Am J Med 2010; 123:e11.

4. American College of Emergency Physicians. Emergency ultrasound guidelines. Ann Emerg Med. 2009;53:550-570.

5. Hall MK, Taylor RA, Luty S, Allen IE, Moore CL. Impact of point-of-care ultrasonography on ED time to disposition for patients with nontraumatic shock. Am J Emerg Med 2016;34:1022-1030.

6. Mjolstad OC, Dalen H, Graven T, Kleinau JO, Salvesen O, Haugen BO. Routinely adding ultrasound examinations by pocket-sized ultrasound devices improves inpatient diagnostics in a medical department.Eur J Intern Med. 2012; 23:185-191.

7. Whitson MR, Mayo PM. Ultrasonography in the Emergency Department. Critical Care. 2016; 20:227.

8. Mandavia D, Aragona J, Chan L, Chan D, Henderson S. U1trasound Training for Emergency Physicians - A Prospective Study. Acad Emerg Med 2000;7:1008-1014.

9. Neri L, Storti E, Lichtenstein D. Toward an ultrasound curriculum for critical care medicine. Crit Care Med 2007; 35(5 Suppl.): S290-S304. 